\title{
Introducing Energy Efficient Strategies of Virtual Backbone Nodes in Wireless Sensor Network
}

\author{
Kartik Kumar \\ Chang Gung University \\ Guishan Dist., \\ Taoyuan City 33302, Taiwan
}

\author{
Lokesh Sharma \\ Chang Gung University \\ Guishan Dist., \\ Taoyuan City 33302, Taiwan
}

\begin{abstract}
Wireless Sensor Network is a Research issue in which construction of Virtual Backbone schemes over multi-hop wireless sensor networks. This paper investigates the problem of connectivity for random placement of nodes by minimizing active sensor nodes. Several backbone construction techniques have been reported in recent years regarding the routing problem of the ad hoc wireless sensor networks. As the network size is growing in the physical world, redundancy in nodes also increased because of this the redundancy it affects the WSN performance. Hence the classification of all these backbone construction techniques is done, which are based on the connected dominating set (CDS) and other techniques. A generalized scheme to be more robust, having lesser number of Backbone nodes with higher mobility is proposed.
\end{abstract}

\section{Keywords}

Wireless Sensor Network, Sensor Network, Backbone Construction using Cluster Head in Spanned Forest (BC2HF), Maximum Degree Spanned Forest (MDSF)

\section{INTRODUCTION}

In recent years, advances in miniaturization, however straightforward low power circuit design and enhanced minimal effort, small size batteries have made a new technological vision possible: wireless sensor network (WSN). These networks combine wireless communication and minimal computation facilities with the sensing of physical phenomenon which can be easily embedded in the physical environment [1]. In other words, a sensor node is fundamentally a device that changes over a detected attribute (such as temperature, vibrations) into a form understandable by the users. WSNs, which can be considered as a special case of ad-hoc networks with reduced or no mobility, are relied to expand the deployment in coming years, as they enable reliable monitoring and analysis of unknown and untested environments[3]. These networks are "data-centric", i.e., unlike traditional ad-hoc networks where information is requested for a particular node, information is asked based on certain attributes such as, "Which area has temperature more than $35 \mathrm{C}$ or $95 \mathrm{~F} "$. Consequently A huge quantities of sensors need to be deployed to accurately reflect the physical attribute in a given range. Because of absence of a superior word, typical sensor consists of transducer to sense a given physical quantity with a predefined exactness, an embedded processor for local, small memory unit for storage of data and remote handset to transmit or get data and all these devices run on the power supplied by an attached battery.

In many case, there are numerous essential sensors transducers that could convert many problems quantities such

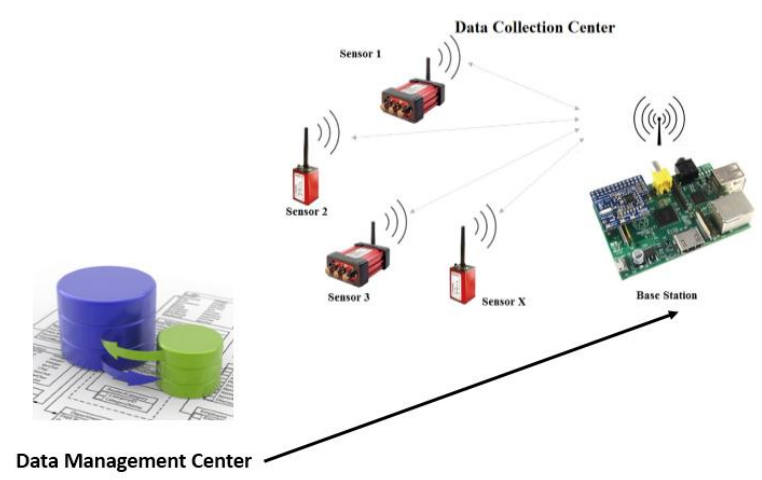

Fig 1: WSN Diagram

as temperature, pressure, velocity, acceleration, stress and strain, fatigue, tilt, light intensity, sound, humidity, gas sensors, many others .These basic generic transducers need to be interfaced and connected to other devices and such specially designed unit can be utilized for a given particular application. A wireless sensor network (WSN) consists of a large number of sensor nodes (SN's) and exploring their best possible use is a challenging problem. As the objective of a $\mathrm{SN}$ is to monitor some physical quantity in a given area, the sensor needs to be deployed with adequate density so that sensing of the complete region should be done, without leaving any void or unsensed area as shown in Fig. 3.

It is interesting to note that exact particulars of different components, may depend on the type of applications in hand, but the basic characteristics are essentially present to fulfill

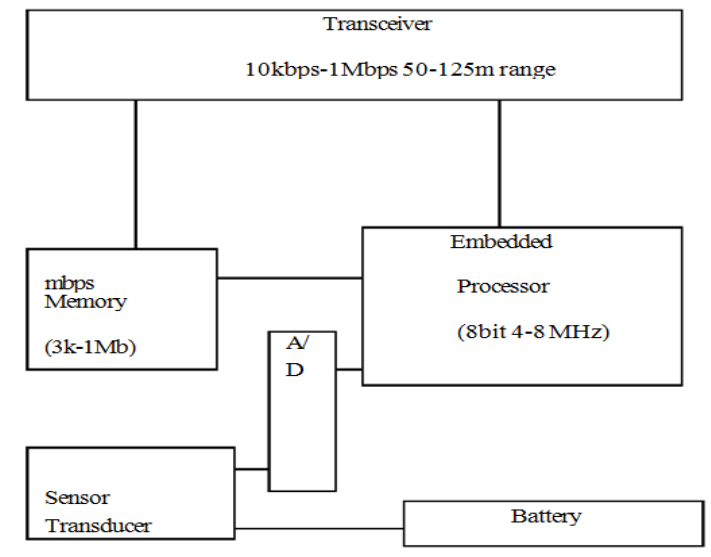

Fig 2: Functional block diagram of a typical Sensor Node 


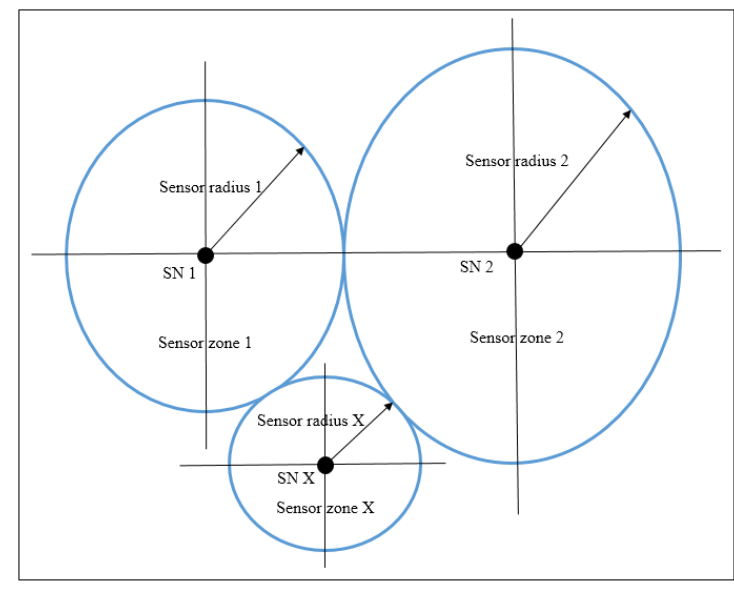

Fig 3: Sensing and Communication range of SNs

Desired application functionalities. There are few integrated sensors commercially accessible and can be utilized straightforwardly as plug and play unit to monitor and control some specific physical parameters as decided by the user.

In other words, given the sensing range of each $\mathrm{SN}$ and the area to be covered, an adequate number of SNs should be needed to be placed throughout the area so that no corner is left out. The SNs can be placed deterministically at prespecified locations or could be distributed randomly .so, if $\mathrm{N}$ SNs are put in an area $\mathrm{A}=\mathrm{L} * \mathrm{~L}$, then the SN's density can be given by $\lambda=\mathrm{N} / \mathrm{A}$. The sensing range rs, of each sensor, has its own sensing range and to cover the whole Space, adjacent SNs need to be located close to each other and at most at a distance of 2 rs from each other as illustrated by three positions of SN2, SN2', SN2" with different overlapping areas between SN1 and SN2.If the SN2 are uniformly distributed with the node density of $\lambda$, the probability that there are some $\mathrm{m} \mathrm{SNs}$ within the space $\mathrm{S}$ is poisons distributed as:

$$
P(m)=(\lambda S)^{m} \times e^{-\lambda s}
$$

Where space $\mathrm{S}=\pi \mathrm{r} 2 \mathrm{~s}$ for two dimensional spaces. Transmission between adjacent SNs using the wireless transceiver and is feasible only when there is at least one SN within the communication scope of every SN. In this way, not only the detecting scope, but the communication connectivity is at least equally important so that sensor data could be received by another sensor network. it may be noted that the data from a single $\mathrm{SN}$ is not adequate to make any useful decision and data need to be collected from a set of SNs in arriving at an intelligent interpretation.

So the real question is how far away the SNs can be located. As illustrated in two SNs can be separated by 2rs distance from sensing coverage point of view. Considering this to bean allowed maximum distance between two adjacent sensors SN1 and SN2. To enable a data transfer between them. The minimum allowed communication distance should be 2 rs. This infers the wireless communication scope of a sensor must be at least twice the sensing distance and is the minimum distance to ensure connectivity between sensing devices to communicate with each other.

\section{APPLICATION}

Thousands of sensors over strategic locations are used in structure such as an automobile or an airplane, so that conditions can be constantly monitored both from inside and the outside and a real-time warning can be issued whenever a major problem is approaching in the checked element, these wired sensors are large to cover as much area is desirable. The organization of such a system ought to be pre-planned to locate a strategic position to place these nodes and then should be installed appropriately. Wireless sensor network has been conceived with military application in battlefield surveillance and tracking of adversary exercises. There are endless application do exist for sensor network such as weather monitoring, security, and strategic observation, disseminated figuring, fault detection and diagnosis in machinery, large bridges and tall structures, distinguishing ambient conditions, for example, temperature, movement, sound, light ,radiation , vibration, smoke, gasses or the nearness of certain natural and chemical objects.

\section{CHALLENGES}

Ad hoc deployment - Sensors nodes are randomly deployed which requires that the system is able to cope up with the resultant distribution and form connections between the nodes. Furthermore, the system should be adaptive to changes in network connectivity as a result of node failure.

Computational capabilities - Sensors nodes have limited computing power and therefore may not be able to run sophisticated network protocols prompting light weighted and basic forms of routing protocols.

Energy consumption without losing accuracy - Sensors nodes can use up their limited energy supply carrying out computations and transmitting information in a wireless environment .As such energy - conversing forms of communication and computation are crucial as the node lifetime demonstrates a solid reliance on the battery lifetime. In a multi- hop WSN, nodes play a dual role as data sender and data router.

Scalability - The number of sensor nodes deployed in the sensing area may be in the order of hundreds, thousands or more. Any routing plan must be sufficiently adaptable to respond to events and capable of operating with such large number of sensor nodes. Most of the sensor can remain in the sleep state until an event occurs with data from just a couple remaining sensors giving a coarse quality.

Communication range - The bandwidth of the wireless links connecting sensors nodes is often limited, hence constraining inter-sensor communication .Moreover, limitations on energy forces sensors node to have short transmission ranges.

Fault tolerance - Some sensor nodes may fail or be blocked due to absence of power, physical harm or environmental interference. If many nodes fail, MAC and routing protocols must accommodate formation of new links and route to the data collection BSs. Therefore multiple levels of redundancy may be needed in a fault tolerant WSN.

Connectivity - High node density in sensor network precludes them from being completely isolated from each other. In this manner sensor hubs are required to be highly connected .In addition, connectivity depends on the, possibly random distribution of nodes.

Transmission media - In a multi-hop sensor network, communicating nodes are linked by a wireless medium. In general bandwidth requirements of the sensor, applications will be low, in order of $1-100 \mathrm{~kb} / \mathrm{s}$.

QoS - In some applications, the data should be delivered within a certain period of time form the moment it is sensed, otherwise data will be useless. 
Control overhead - When the number of retransmission in wireless medium increases because of collisions, the latency and vitality utilization too increases. Hence, control packet overhead increases linearly with the node density.

Security - Security is an important issue which does not mean physical security, but it implies that both verification and encryption ought to be achievable .But with limited resources implementation of any complex algorithm should be stayed away from. Subsequently a tradeoff exists between the security level and energy consumption in a WSN.

\section{DESIGN ISSUES}

The advancement in technology has made it possible to have a network of 100 s or even thousands of extremely small, low powered devices equipped with programmable computing, multiple parameter sensing, and wireless communication capability, enhancing the reliability, accuracy of data and the coverage area. Some of the advantages of WSN over wired ones are as follows:-

Extended range: one huge wired sensor can be replaced by many smaller wireless sensors of the same cost, such a macro sensor can sense only a limited region whereas a network of the smaller sensor can be distributed over a wide range.

Fault Tolerant: with macro sensors, the failure of one node makes that area completely unmonitored till it is replaced. At most the accuracy of data collected may be somewhat reduced.

Mobility: Wireless sensor are equipped with the battery they can possess limited mobility, thus if a region becomes unmonitored, the nodes rearrange themselves to distribute evenly i.e., these nodes can be made to move towards the area of interest but having lower mobility as compared to ad hoc networks. The Wireless Sensor Network has gained popularity in late decades. It consists of the nodes which are battery operated devices, processors, and sensors. As nodes are driven by battery worked devices it is obvious the time will come when the battery will get discharged and the node will go down permanently [7]. That's why in the design of the Wireless Sensor Network it is very crucial to saving the energy at every node by putting the node into a sleep state when the node is idle. As the nodes contain the processor and sensors also with a battery the state transition of the processor and sensors takes negligible energy. The nodes are eating up $60 \mathrm{~mW}, 45 \mathrm{~mW}, 45 \mathrm{~mW}, 90 \mathrm{~mW}$ of energy while transmission, reception, listening and sleeping periods respectively [1]. When numerous nodes present inside the WSN then it is more suitable to form those nodes inside one cluster so all the nodes in the cluster are easy to manage rather than to manage each and every node individually. The cluster is the main piece of cake which communicates with the third party. This third party may be a base node, controller node, another cluster head, router etc [4][5]. Here as written the energy consumption during the sleep time is $90 \square \mathrm{mW}$ so it is better to put the idle node into sleep mode to minimize the power consumption and improves the battery life [8][13]. When the connection is shared between the various nodes at this time at a time only one node has the permission to send the data to the other node for that it must be made sure that the channel or link is free or not. This should be possible with the assistance of sending the request to send signal referred as rts to the receiver node and get back clear to send denoted as cts from the receiver side to the sender [14]. For this kind trading the signs between two nodes, both the nodes has to communicate with each other. By doing this kind of communication each nodes may get data about their neighbor nodes with which the particular node is connected specifically. This procedure of correspondence between two nodes of exchanging information is referred as Local Gossips [15]. This paper proposes the scheduling algorithm of the nodes by putting them into sleep mode when they are idle based on data produced by the adjacency matrix and the energy consumption is to be considered during the time of transmitting and receiving only, not during the idle listening at the time of communication-based on the shortest depth.

\subsection{Energy Consumption}

Minimizing the energy consumption of WSs is critical yet a challenge for the design of WSNs. The energy utilization for WSN involves three different components: a sensing unit, communication unit and computing /processing unit. In order to converse energy, some SNs go to sleep mode and need to consider energy consumed in that state.

Sensing transducer - Sensing transducer is responsible for capturing the physical parameters of the environment. Its essential capacity is to do physical signal sampling and convert into electrical signals. The energy consumption of this part depends on the hardware and the application and sensing energy is only a small part of the total energy consumption.

A/D convertor - An A/D convertor for the sensor consumes only $3.1 \mu \mathrm{W}$, in $31 \mathrm{pJ} / 8$-bit sample at 1 volt supply. The standby power consumption at $1 \mathrm{v}$ supply is $41 \mathrm{pW}$.

Assuming the $\mathrm{D} / \mathrm{C}$ is not noise limited, the power bound on energy per sample for the successive approximation architecture is roughly computed as:

$$
E_{\text {min }}=C_{\text {total }} * V^{2} \text { ref }
$$

where $C_{\text {total }}$ is the total capacitance of the array including the bottom plate parasites, and $V_{r e f}$ is a common mode input voltage the on which the comparator operates.

Transmission Energy - based on the transmission energy transmits a $\mathrm{K}$ - bit message to distance $\mathrm{d}$ can be computed as:

$$
\begin{aligned}
E_{T X}(k, d) & =E_{T X} \text { elec }(k)+E_{T X}-a m p(k, d) \\
& =E e t e c \times k+\varepsilon \times k \times d^{2}
\end{aligned}
$$

Where $E_{T X}$-elec is the consumption of the transmission electronics energy, $E_{T X}-a m p$ is the transmit amplifier energy consumption.

Receiver Energy - To receive a $\mathrm{K}$ bit message, the energy consumed is:

\section{MOTIVATION}

$$
E R X(k)=E R X-e l e c(k)=\text { Eelec } \times k
$$

As the world is day by day expanding and the congestion over the limited resources is increasing, it is the critical stage to build efficient algorithms so that the resources will be used in a gentle way. Wireless Sensor Network is an area where a lot of research has been taken place regarding the transmission techniques, saving energy techniques which use less energy and retain the network for a long time. The proposed model mainly focuses on the energy efficient model. Previously the techniques are outdated, since they didn't minimize transmission and maximize end to end delivery. They having no concern over Energy-efficient techniques. In the proposed model every node, collects its neighbor information and calculates the depth. After collecting the neighbor information every node sends the neighbor information wise to the controller node via the Cluster head. 
Table 1: List of related work

\begin{tabular}{|c|c|c|c|}
\hline $\begin{array}{l}\text { S. } \\
\text { No }\end{array}$ & Author name, Title, year & Technology used & Results \\
\hline 1. & $\begin{array}{l}\text { Yu Xiang et. Al, A Distributed } \\
\text { Algorithm for Virtual Backbone } \\
\text { Construct ion with Cellular } \\
\text { Structure in WSNs,2012[10] }\end{array}$ & $\begin{array}{l}\text { Distributed Method: Strategic points are } \\
\text { dynamic,1-hop information, satisfying } \\
\text { hexagonal conditions }\end{array}$ & $\begin{array}{l}\text { Distortion in virtual Backbone, unreliable } \\
\text { communication channel }\end{array}$ \\
\hline 2. & $\begin{array}{l}\text { A Uniform Energy Consumption } \\
\text { Algorithm for Wireless Sensor and } \\
\text { Actuator Networks Based on } \\
\text { Dynamic } \quad \text { Polling } \quad \text { Point } \\
\text { Selection,2013[20] }\end{array}$ & Path planning strategies & A path-planning algorithm for the actuator \\
\hline 3. & $\begin{array}{l}\text { Taxing Zhao et. Al., On } \\
\text { Maximizing the Lifetime of } \\
\text { Wireless Sensor Networks Using } \\
\text { Virtual Backbone Scheduling,2012 } \\
\text { [11] }\end{array}$ & $\begin{array}{l}\text { Schedule transition graph: Energy level is } \\
\text { computed, Record energy level after every } \\
\text { round, Largest energy level is considered. }\end{array}$ & Complex in nature \\
\hline 4. & $\begin{array}{l}\text { A virtual queue-based back- } \\
\text { pressure scheduling algorithm for } \\
\text { wireless sensor networks, 2015 } \\
{[21]}\end{array}$ & $\begin{array}{|lr|}\text { Virtual queue-based } & \text { back-pressure } \\
\text { scheduling algorithm: } & \text { Back-pressure } \\
\text { scheduling Delay reduction } & \end{array}$ & $\begin{array}{l}\text { Establishes the gradient } 1^{\text {st }} \text { at each node in a } \\
\text { WSN and integrates this gradient when } \\
\text { calculating the queue backlog differential } \\
\text { between neighboring nodes }\end{array}$ \\
\hline 5. & $\begin{array}{l}\text { An Iterative Local Replacement of } \\
\text { Maximizing the Lifetime of } \\
\text { Wireless Sensor Networks, 2014 } \\
{[19]}\end{array}$ & $\begin{array}{|lr|}\text { Iterative Local Replacement Based } \\
\text { Algorithm: Local Replacement-hop } \\
\text { information }\end{array}$ & Increases message overhead and execution time \\
\hline 6. & $\begin{array}{l}\text { Stefano Basagni et. Al., Using } \\
\text { Multiple Radios for Ad } \mathrm{Hoc} \\
\text { Backbone Construction } \\
\text { Maintenance,2011[12] }\end{array}$ & $\begin{array}{l}\text { M Backs(Network with single Radio } \\
\text { interference): Gateway nodes, Virtual M } \\
\text { Back is implemented }\end{array}$ & More maintenance is required \\
\hline 7. & $\begin{array}{l}\text { Interference in wireless multi-hop } \\
\text { ad-hoc networks and its effect on } \\
\text { network capacity, July } 2004 \text { [18] }\end{array}$ & $\begin{array}{l}\text { MM Backs(Multiple hop mobile network } \\
\text { with Multiple radio interference): Radio } \\
\text { with different ranges }\end{array}$ & $\begin{array}{l}\text { Outperform Backbone size, connectivity time, } \\
\text { resilience to breakage. }\end{array}$ \\
\hline 8. & 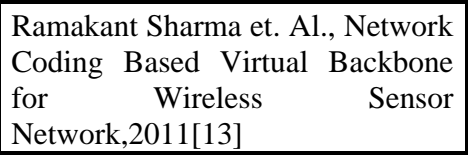 & $\begin{array}{l}\text { NCVB (Network coding based Virtual } \\
\text { Backbone): Encode number of packets as } \\
\text { a single unit. }\end{array}$ & $\begin{array}{l}\text { Reduces total number of transmission, more } \\
\text { efficient, saves lot of energy }\end{array}$ \\
\hline 9. & $\begin{array}{l}\text { Chiranjib Patra et.al.,Designing } \\
\text { energy efficient Topologies for } \\
\text { Wireless Sensor Network : Neural } \\
\text { Approach, } 2010 \text { [14] }\end{array}$ & $\begin{array}{lll}\text { Self-Organizing } & \text { Feature } & \text { Map(SOFM) } \\
\text { network Model: Winning neurons, k- } \\
\text { neighbor algorithm }\end{array}$ & $\begin{array}{l}\text { Low power consumption, synchronization done } \\
\text { for future work }\end{array}$ \\
\hline 10. & \begin{tabular}{lrr|} 
Junchao Ma Et. Al., & Energy \\
Efficient TDMA Sleep Scheduling \\
in $\quad$ Wireless & Sensor \\
Networks,2009[15] & \\
\end{tabular} & $\begin{array}{l}\text { Contiguous Link scheduling: interference } \\
\text { free link scheduling }\end{array}$ & $\begin{array}{l}\text { Avoiding collisions, idle listening and } \\
\text { overhearing }\end{array}$ \\
\hline 11. & $\begin{array}{l}\text { Shio Kumar Singh et. Al. , Energy } \\
\text { Efficient Homogenous Clustering } \\
\text { Algorithm for Wireless Sensor } \\
\text { Networks,2010[16] }\end{array}$ & $\begin{array}{l}\text { Homogenous Clustering Algorithm, Zones } \\
\text { are divided, compress data }\end{array}$ & Extending network Life time \\
\hline 12. & $\begin{array}{l}\text { Samia A. Ali et. Al." Distributed } \\
\text { patching for mobile wireless } \\
\text { sensor networks",2012[17] }\end{array}$ & $\begin{array}{l}\text { EEBBPv1: Disconnected backbones of } \\
\text { MWSNs and/or WSNs when less than } \\
\text { 20\%, ECDS (Expected Area Coverage } \\
\text { Deployment) }\end{array}$ & $\begin{array}{l}\text { Abrupt Stopping is not handled, Depletion of } \\
\text { energy cause large Disconnection }\end{array}$ \\
\hline 13. & $\begin{array}{l}\text { Samia A. Ali et. Al.” Distributed } \\
\text { patching for mobile wireless } \\
\text { sensor networks",2012[17] }\end{array}$ & $\begin{array}{l}\text { EEBBPv2: Disconnected backbones of } \\
\text { MWSNs and/or WSNs when less than } \\
35 \%\end{array}$ & Expends more energy and time \\
\hline
\end{tabular}

The Cluster head node works like a connecting chain between all nodes which are isolated. This model provides the synchronization flexibility in terms of sleep and wakes up scheduling for efficient energy scheme in Wireless Sensor Network.

\subsection{Related Work}

The related work is tabulated below in Table 1 .

\subsection{Proposed Work}

To design Backbone construction, firstly develop a Cluster Regions (CR) in this an adapting technique Maximum Degree 
Spanned Forest (MDSF) is used to make Cluster Region which is an individual Forest, and there is no connectivity among those regions. In MDSF the node to be picked are the nodes which are having the maximum degree and occupies the maximum area in the region. Once the number of nodes is specified in a Cluster Region (MDSF), the spanning trees are formed among those nodes, now it is a small backbone. And followed by that, once regions are defined than the next technique is named as Backbone.

The Construction using Cluster Head in Spanned Forest (BC2HF) will construct Backbone Construction. In this, the algorithm searches for cluster Head which is most connectable with other forests, once all the forest connects each they will be a network and the objective will be fulfilled.

\section{Maximum Degree Spanned Forest (MDSF)}

- Select Network Region of Size $x^{*} y$, define the values of communication range, sensing range etc.

- Input the number of nodes and by using Random Distribution, it's spread it over the region.

- $\quad$ Select a sensor node which having maximum degree among all (Fig 6).

- Keep that node as the Cluster Head node which provides inter-connectivity between different forests.

- Once first cluster region developed, start iterating same steps and design number of the forest.

- Now the region is filled with a number of Forests.

- Select First Cluster Region (CR) and by the help of the scheme, it is decided which will be the Cluster Head $(\mathrm{CH})$ and having connectivity with other Forest $\mathrm{CH}$.

- Do the same step with all other Forest.

- Now there is connectivity between all the forest and Backbone is constructed (Fig 7).

\subsection{Flow Chart}

In the given flow diagram, firstly numbers of nodes are introduced and their communication as well as transmission range. By the help of algorithm select a node that having a maximum degree and put that node in a set of the cluster head. Now define the region of cluster head and sleep rest of the nodes so that maximum efficiency will be attained. Do the same process for all the cluster head nodes and produce so many forests in the given network. Join the forest by applying any interconnectivity algorithm between two cluster head. Fig 8 shows the flow diagram.

\subsection{MatLab Results}

In Fig 8 the first diagram shows the random deployment of nodes in a region, whereas the second diagram displays how the cluster head is made and it also shows its responsibility of doing communication with rest of the nodes in region and the last graph shows how the interconnectivity took place between different cluster heads in a network.

\section{CONCLUSION}

A generalized scheme to be more robust, having lesser number of Backbone nodes with higher mobility is proposed, which is an energy efficient technique for WSN. Based on BC2HF Construction using Cluster Head in Spanned Forest will construct Backbone Construction and the MDSF algorithm searches for cluster Head which is most connectable with other forests, once all the forest connects each they will be a network and the objective will be fulfilled. Finally, an efficient way of Backbone Construction using Cluster Head in Spanned Forest (BC2HF) and connectivity between all the forest and backbone is constructed.

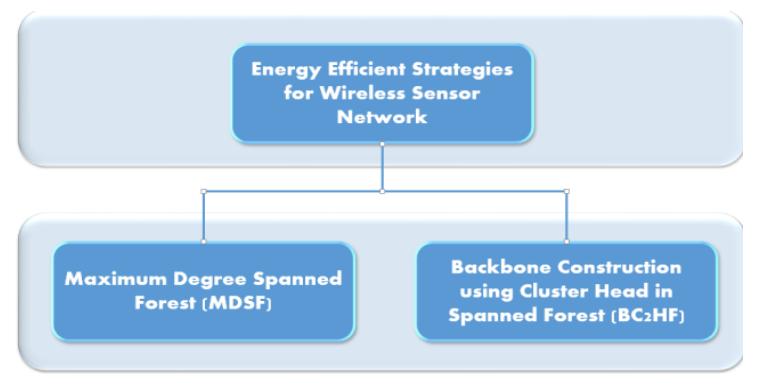

Fig 4: Functional block diagram of a typical Sensor Node
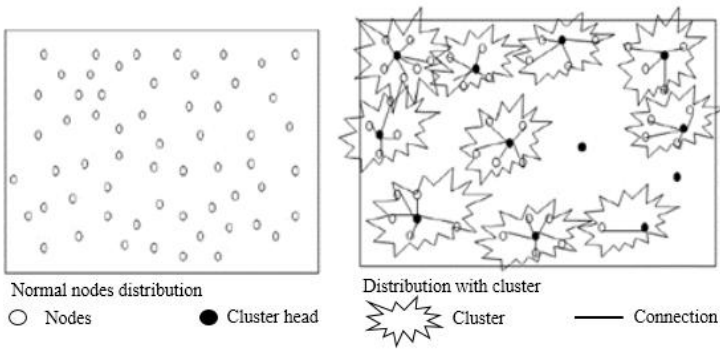

Fig 5: Backbone Construction using Cluster Head in Spanned Forest (BC2HF)

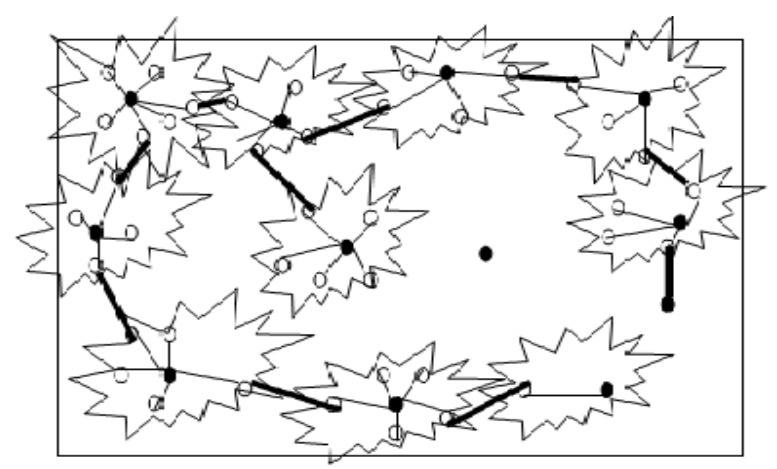

Fig 6: Connectivity between all the forest and Backbone is constructed

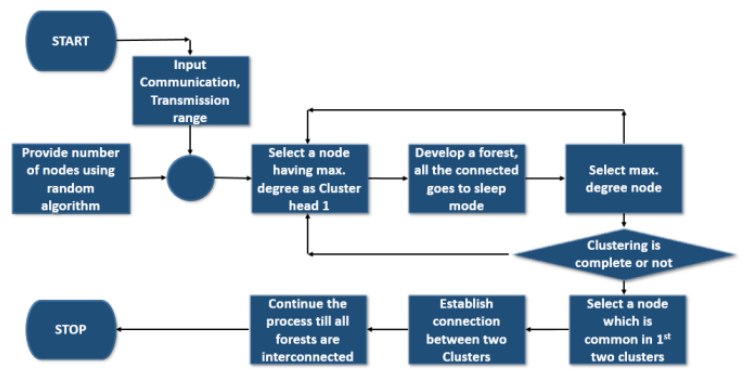

Fig 7: The forest by applying any interconnectivity algorithm between two cluster head. 

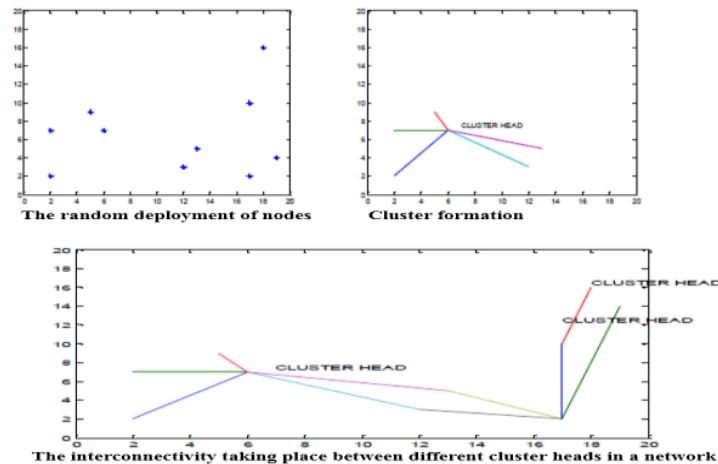

Fig 8: Nodes deployment to cluster formation and finally cluster interconnectivity

\section{REFERENCES}

[1] Liu and Yanwei Wu, Xiang-Yang Li, Yun Hao Wei Lou, (2010) "Energy-Efficient Wake-Up Scheduling for Data Collection and Aggregation", IEEE TRANSACTIONS ON PARALLEL AND DISTRIBUTED SYSTEMS, vol. 21, no. 2, pp 275-287.

[2] Sung-Yeop Pyun; Dong-Ho Cho, "Energy-Efficient Scheduling for Multiple-Target Coverage in Wireless Sensor Networks," Vehicular Technology Conference (VTC 2010-Spring), 2010 IEEE 71st, vol., no., pp.1,5, 16-19 May 2010

[3] Dasgupta, S.; Bhattacharya, I.; Bose, G., "Energy-aware cluster based node scheduling algorithm in wireless sensor network for preserving maximum network life time," Methods and Models in Computer Science, 2009. ICM2CS 2009. Proceeding of International Conference on , vol., no., pp.1,7, 14-15 Dec. 2009

[4] Ying Lin; Xiao-Min Hu; Jun Zhang; Ou Liu; Hai-lin Liu, "Optimal node scheduling for the lifetime maximization of two-tier wireless sensor networks," Evolutionary Computation (CEC), 2010 IEEE Congress on , vol., no., pp. $1,8,18-23$ July 2010 .

[5] Jennifer Yick, Biswanath Mukherjee and Dipak Ghosal, (2008) "Wireless sensor network survey" ,ELSEVIER, pp 2292-2330.

[6] Rick W. Ha , Pin-Han Ho , X. Sherman Shen, Junshan Zhang, (2006) "Sleep scheduling for wireless sensor networks via network flow model",ELSEVIER, pp 24692481.

[7] Anandamurugan, S.; Venkatesh, C., "SSP: A Sleep Scheduling Protocol for improving the lifetime of wireless sensor networks," Control, Automation, Communication and Energy Conservation, 2009. INCACEC 2009. 2009 International Conference on, vol., no., pp.1,6, 4-6 June 2009.

[8] I. Marı'n, J. Arias, E. Arceredillo, A. Zuloaga, I. Losada and J. Mabe, (2007) "LL-MAC: A low latency MAC protocol for wireless self-organised etworks"ELSEVIER:Microprocess and Microsystems",pp 197-209.

[9] Suriyachai, P.; Roedig, U.; Scott, A., "A Survey of MAC Protocols for Mission-Critical Applications in Wireless Sensor Networks," Communications Surveys \& Tutorials, IEEE, vol.14, no.2, pp.240,264, Second Quarter 2012.
[10] Yu Xiang, Xiaojuan Liu, Yong Tang, Juncong Xiao, and Jun Zhang, "A Distributed Algorithm for Virtual Backbone Construct ion with Cellular Structure in WSNs" International Journal of Distributed Sensor Networks,2012.

[11] Yaxiong Zhao; Jie Wu; Feng Li; Sanglu Lu, "On Maximizing the Lifetime of Wireless Sensor Networks Using Virtual Backbone Scheduling," Parallel and Distributed Systems, IEEE Transactions on, vol.23, no.8, pp.1528,1535, Aug. 2012

[12] Basagni, S.; Nanni, M.A., "Using Multiple Radios for Ad Hoc Backbone Construction and Maintenance," Mobile Adhoc and Sensor Systems (MASS), 2011 IEEE 8th International Conference on , vol., no., pp.170,172, 17 22 Oct. 2011

[13] Ramakant Sharma et. Al., "Network Coding Based Virtual Backbone for Wireless Sensor Network" (IJCSIT) International Journal of Computer Science and Information Technologies, Vol. 3 (3) , 2012,4341 4344.

[14] Chiranjib Patra,Anjan Guha Roy ,Samiran Chattopadhyay and Parama Bhaumik, "Desig ning Energy-Efficient Topologies for Wireless Sensor Network: Neur al Approach", International Journal of Distributed Sensor Networks,2010.

[15] Junchao Ma; Wei Lou; Yanwei Wu; Mo Li; Guihai Chen, "Energy Efficient TDMA Sleep Scheduling in Wireless Sensor Networks," INFOCOM 2009, IEEE, vol., no., pp.630,638, 19-25 April 2009 doi: 10.1109/INFCOM.2009.5061970

[16] Shio Kumar Singh ,M P Singh and D K Singh, "Energy Efficient Homogenous Clustering Algorithm for Wireless Sensor Networks", International Journal of Wireless \& Mobile Networks ( IJWMN ), Vol.2, No.3, August 2010

[17] Samia A. Ali,Khaled M.Shaaban, Islam M Alkabbany,"Distributed patching for mobile wireless sensor networks",Elsevier Journal of Network and Computer Applications ,2012.

[18] R. HEKMAT and P. VAN MIEGHEM, "Interference in Wireless Multi-Hop Ad-Hoc Networks and Its Effect on Network Capacity", Wireless Networks 10, 389-399, 2004 Kluwer Academic Publishers. Manufactured in The Netherlan, 2004

[19] C.H.T.G.Ramya and E. Nagarjuna, "An Iterative Local Replacement of Maximizing the Lifetime of Wireless Sensor Networks", IJCSIET--International Journal of Computer Science information and Engg., 2004.

[20] Shuo Li,1 Jun Peng, Weirong Liu, Zhengfa Zhu, and Kuo-Chi Lin, "A Uniform Energy Consumption Algorithm for Wireless Sensor and Actuator Networks Based on Dynamic Polling Point Selection",NCBI, Sensors (Basel) 14(1): 95-116. Published online 2013 Dec 19. doi: 10.3390/s140100095. Jan 2014.

[21] Zhenzhen Jiao, Baoxian Zhang, Wei Gong and Hussein Mouftah," A virtual queue-based back-pressure scheduling algorithm for wireless sensor networks ", EURASIP Journal on Wireless Communications and Networking,2015. 